\title{
The effects of poloxamer 188 on the autophagy induced by traumatic brain injury
}

Haijun Bao ${ }^{\mathrm{a}}$, Xiaofang Yang ${ }^{\mathrm{a}}$, Ying Zhuang ${ }^{\mathrm{a}}$, Yuxiu Huang, ${ }^{\mathrm{a}}$, Tao Wang ${ }^{\mathrm{b}}$, Mingyang Zhang,

Dingkun Dai ${ }^{\mathrm{c}}$, Shaoxian Wang ${ }^{\mathrm{a}}$, Xiao Hua ${ }^{\mathrm{a}}$, Gengping Huang ${ }^{\mathrm{a}}$, Jinxia Kuai ${ }^{\mathrm{d}, ~ *}$, Luyang Tao ${ }^{\mathrm{b}, ~ *}$

${ }^{\mathrm{a} D e p a r t m e n t ~ o f ~ P a t h o l o g y, ~ X u z h o u ~ M e d i c a l ~ U n i v e r s i t y, ~ X u z h o u, ~ C h i n a . ~}$

${ }^{\mathrm{b}}$ Department of Forensic Medicine, Soochow University, Suzhou, China.

${ }^{\mathrm{c}}$ Department of Forensic Identification, The People's Hospital of Subei, Yangzhou, China.

${ }^{\mathrm{d}}$ Department of Forensic Medicine, Xuzhou Medical University, Xuzhou, China.

All the authors contributed equally to this work.

*Corresponding author: Jinxia Kuai and Luyang Tao

Email: 55752663@163.com, taoluyang@ suda.edu.cn

Tel: 086-516-85748428

Department of Forensic Medicine, Xuzhou Medical University, Xuzhou, China

Add: 209 Tongshan Road, Xuzhou, China

Abbreviations: TBI, traumatic brain injury; P188, poloxamer 188; CCI, controlled cortical impact 


\begin{abstract}
Poloxamer 188 (P188) has been reported to reseal plasma membranes and attenuate TBI-induced neuronal death by suppressing apoptosis. Recent studies also confirm increased autophagy after traumatic brain injury (TBI). The present study aimed to investigate the effects of plasmalemmal resealing by P188 on neuronal autophagy in TBI. Scratch test was performed in rat cell line PC-12 in vitro, followed by immunofluorescence analysis of LC3 $24 \mathrm{~h}$ after PC-12 cell stretch-injury in vitro. CD1 mice were randomized into saline and P188-treatment groups (both undergoing intravenous injection of $4 \mathrm{mg} / \mathrm{ml}, 100 \mu \mathrm{l}$ via the caudal vein $30 \mathrm{~min}$ after TBI) as well as sham group. To analyze the effect of P188 on autophagy, the LC3 protein levels were assessed by western blotting $1 \mathrm{~h}, 6 \mathrm{~h}, 12 \mathrm{~h}, 24 \mathrm{~h}$, and $48 \mathrm{~h}$ after TBI. The autophagy-associated protein levels of Beclin-1, Bcl-2, and p62 were likewise determined. In vitro, the scratch test showed that the wound healing rate was significantly improved at $12 \mathrm{~h}$ and $24 \mathrm{~h}$ in P188 groups, and LC3 immunofluorescence analysis indicated that P188 induced extensive formation of LC3 puncta in PC-12 cells. In vivo, western blotting analyses revealed elevations of the LC3-II/LC3-I and Beclin-1/bcl-2 ratios as well as downregulation of $\mathrm{p} 62$ in the saline group, in contrast with the more significant increases of LC3-II/LC3-I and Beclin-1/bcl-2 ratios and the further downregulation of p62 in P188-treated group. These results revealed that plasma membranes were resealed after TBI, in which P188 aggravated autophagy in vivo.
\end{abstract}

Key words: Traumatic brain injury, Poloxamer 188, Autophagy 


\section{Introduction}

Traumatic brain injury (TBI) typically contributes to the disintegrity of cell membrane and threatens neuronal survival $[9,18]$. However, poloxamer 188 (P188) is an amphiphilic polyethylene-polypropylene-polyethylene copolymer, characterized by its properties in sealing membranes and restoring the plasma membrane integrity of neurons following membrane injury $[8,13,16]$.

Autophagy leads to degradations of long-lived proteins, aggregated proteins and damaged organelles, and ultimately the maintenance of material recycling and homeostasis $[3,10]$. There have been controversies regarding the genesis of autophagy, such as the formation of double-membrane structures that engulf cytoplasmic materials and fuse with lysosomes, or consequence of hypoxia, nutritional deprivation, or exposure to radiation, chemicals and other stimulants [3, 10]. The microtubule-associated protein 1 light chain 3 (LC3) is a biomarker in biochemical assays at autophagosomal levels, with the conversion of endogenous LC3-I to LC3-II indicating the extent of autophagy [3, 10]. Bcl-2 can reportedly regulate autophagy via its interaction with Beclin-1. As a component of the main signal-initiating complex (class III PI3 kinase, Beclin-1 and p150 protein), Beclin-1 is one of the key proteins regulating autophagy and triggering a cascade of proteins involved in the formation of autophagolysosomes $[3,10]$. Autophagy plays multifunctional roles in the maintenance of cellular homeostasis. Autophagy is an evolutionarily conserved procedure that leads to the degradation of proteins or entire organelles in cells subjected to stress $[3,10]$.

In this study, P188 was used as a cell membrane sealing agent to determine whether plasmalemmal resealing could affect autophagy in neurons after TBI. The previous study and our experiment have found that $\mathrm{P} 188$ restores the intactness of the plasma membrane and attenuates TBI-induced BBB disruption, brain edema and neural cell apoptosis in vivo $[1,18]$. At present, cell line PC12 has been used as an in vitro model to mimic in vivo studies such as neuronal apoptosis, necrosis and autophagy. Cell line PC12 is considered a valid in vitro neuronal model to study the 
mechanisms of neuronal injury as depicted in TBI $[2,5]$.

\section{Methods}

\subsection{TBI model and drug administration}

Adult male CD1 mice (20-25g, $n=66$ ) from the Experimental Animal Center, Xuzhou Medical University were anesthetized under $4 \%$ chloral hydrate $(0.4 \mathrm{mg} / \mathrm{g})$ and mounted in a Kopf stereotactic apparatus. All experimental procedures were in compliance with the NIH Guide for the Care and Use of Laboratory Animals and approved by the Institutional Animal Care and Use Committee of Xuzhou Medical University. Mice were randomly assigned to saline, P188 treated, and sham groups after TBI $(n=6)$. The protein expression was detected at $1 \mathrm{~h}, 6 \mathrm{~h}, 12 \mathrm{~h}, 24 \mathrm{~h}, 48 \mathrm{~h}$ post TBI. Mice were subjected to TBI in the left hemisphere of brain using a drop-weight apparatus, in which a $40 \mathrm{~g}$ counterpoise was dropped from 2-mm-diameter footplate $20 \mathrm{~cm}$ above onto the dura mater with a controlled depth of $1.0 \mathrm{~mm}$, as previously described $[9,14]$. The reproducibility and consistency of this TBI model were ensured by the accurate location and controlled strength, depth and duration of the impact. The subsequent craniotomy and immediate scalp closure did not significantly affect physiological parameters. In addition, $100 \mu \mathrm{l}$ P188 $(4 \mathrm{mg} / \mathrm{ml}$, ScienceLab, USA; Pluronic ${ }^{\circledR}$ F68, Av. M.W. 8400) dissolved in normal saline was intravenously injected via the caudal vein.

Sham-operated mice, which underwent the craniotomy without the impact injury, also received the administration of $100 \mu \mathrm{P} 188$ or normal saline (vehicle) by the caudal vein. For western blotting, mice were likewise injected with P188 (4 mg/ml, $100 \mu \mathrm{l})$ or saline $(100 \mu \mathrm{l}) 30 \mathrm{~min}$ after TBI and sacrificed $1 \mathrm{~h}, 6 \mathrm{~h}, 12 \mathrm{~h}, 24 \mathrm{~h}$ and $48 \mathrm{~h}$ after TBI. Tissue samples were obtained from injured cortex $(2 \times 2 \times 2 \mathrm{~mm}$ tissue block including the impact site and surrounding tissue) and hippocampus (the entire ipsilateral hippocampus including the impact site and surrounding tissue) for western blotting analyses.

\subsection{Western blotting analyses}


The LC3 and p62 levels, and Beclin-1/Bcl-2 ratios were determined by western blotting as from $1 \mathrm{~h}$ till $48 \mathrm{~h}$ after TBI to detect the levels of autophagy in vivo. For western blotting analysis, the injured cortical and hippocampal samples were isolated and handled as previously described [1]. $30 \mu \mathrm{g}$ protein from each sample underwent electrophoresis on a 10\% SDS-PAGE gel on a constant direct current. Proteins were transferred to polyvinylidene fluoride membranes on a semidry electrotransfer unit (Bio-Rad) and incubated with primary antibodies against LC3 (Abcam, ab51520, 1:6000) Beclin-1 (Santa Cruz Biotechnology, sc-11427, 1:1000), Bcl-2 (Millipore, 04-436, 1:1000), and p62 (Abcam, ab51416, 1:8000) in Tris-buffered saline containing $0.1 \%$ Tween-20 (TBST) and 5\% skim milk overnight at $4{ }^{\circ} \mathrm{C}$. Thereafter, the membranes were rinsed thrice and incubated with horseradish peroxidase-conjugated secondary antibody in TBST for $2 \mathrm{~h}$. The PageRulerTM Prestained Protein Ladder (Thermo, 26617, $5 \mu \mathrm{l}$ ) was applied according to the manufacturer's instructions. The immunoreactivity was detected using enhanced chemoluminescent autoradiography (ECL kit, Amersham), according to the manufacturer's instructions. The membranes were stripped and reprobed between the different primary antibodies, as well as with GAPDH (Santa Cruz Biotechnology, sc-166545, 1:1000). The signal intensity of the primary antibody binding was quantified using Quantity One (Bio-Rad) and normalized to the loading control, GAPDH.

\subsection{Cell culture}

To further confirm the effects of P188 and the underlying protective mechanism, we performed the scratch test with PC-12 in vitro. PC-12 cells (ATCC, CRL-1721) were cultured at $37^{\circ} \mathrm{C}$ in DMEM at $5 \% \mathrm{CO}_{2}$ containing $5 \%$ fetal calf serum, and $1 \%$ penicillin/streptomycin (Gibco, Carlsbad, CA). PC-12 cells ( $4 \times 106$ cells) were seeded on $14 \mathrm{~cm}$ petri dishes coated with $50 \mu \mathrm{g} / \mathrm{mL}$ poly-D-lysine (Sigma-Aldrich, St. Louis, MO, USA) and cultured in $20 \mathrm{ml}$ DMEM containing 2\% serum for 3 hours, followed by addition of nerve growth factor (NGF, RD, 556-NG-100) to reach a final concentration of $50 \mathrm{ng} / \mathrm{ml}$. For culture of PC-12 cells in 2\% serum-containing DMEM, 
cells $\left(6 \times 10^{6}\right)$ were seeded on $14 \mathrm{~cm}$ petri dishes (Nunc) coated with $100 \mu \mathrm{g} / \mathrm{ml}$ poly-D-lysine (Sigma). A cell injury controller was employed to stretch cells (Commonwealth Biotechnologies) in saline and P188 treatment groups to produce moderate injury, with P188 $\left(10^{-4} \mathrm{M}\right.$ in PBS) added in the treatment group.

\subsection{Scratch test}

The scratch test mimics cell proliferation during wound healing in vivo. First, a "wound" in a cell monolayer was created, with images captured as from the onset of cell proliferation to the wound closure at regular intervals, followed by image comparison to quantify the wound healing rate of the cells.

The PC-12 cells were seeded at a density of $4 \times 10^{6}$ cells/well on 12 -well plates. On the next day, three straight scratches were made with a $200-\mu 1$ pipette tip in each well, which was then rinsed with PBS, and was replenished with regular media, with the cells observed by light microscopy at $0 \mathrm{~h}, 6 \mathrm{~h}, 12 \mathrm{~h}$ and $24 \mathrm{~h}$.

\subsection{Immunofluorescence staining analysis}

Briefly, subsequent to PC12 cell injury, the cover slips were fixed with $4 \%$ paraformaldehyde in PBS for 20 min, permeabilized with $0.1 \%$ Triton X-100 in $0.1 \mathrm{M}$ PBS for 5 min., blocked in 10\% goat serum for $30 \mathrm{~min}$ and incubated overnight at $4^{\circ} \mathrm{C}$ with polyclonal antibodies against LC3 (Abcam, ab51520, 1:6000, USA). Having been rinsed thrice with $0.1 \mathrm{M}$ PBS ( $\mathrm{pH} 7.4$ ), the cells were incubated with Dylight 488 Affini Pure secondary antibodies (EarthOx, 1:200, USA) for $60 \mathrm{~min}$ at room temperature (r/t) and observed under a Nikon fluorescence microscope (Nikon, Tokyo, Japan).

\subsection{Statistical analyses}

The data are presented as the mean \pm standard error (SE) for all mice in experimental and sham groups. Immunofluorescence staining were analyzed by the One-way ANOVA with Dunnett $t$-test. The statistical methods used for western blot analysis were Two-way ANOVA with Dunnett $t$-test. The statistical analysis was 
performed using the appropriate functions in SPSS 13.0, with differences at $P<0.05$ considered statistically significant.

\section{Results}

\subsection{Determination of autophagy-associated proteins by Western blot after TBI}

To elucidate the effects of P188 on TBI-induced autophagy, the determination of expressions of LC3II and LC3I after TBI was conducted in vivo, which revealed a significantly increased LC3II/LC3I ratio (Fig. 1A, B, C; P<0.05).

Recently, as a specific substrate degraded by autophagy [3], p62 has been reported to interact with ubiquitinated proteins and LC3, ultimately regulating the selective autophagic clearance of protein aggregates [3]. Herein, we assessed the p62 expression levels in the treatment group in comparison with the saline group in vivo. Acute downregulation of p62 expressions were evidenced in the injured cortex (Fig. 1D \& E) and hippocampus (Fig. 1D and F) as from 12-48h after TBI in P188-treated mice. All the above data indicated that autophagy was activated by P188 after TBI in vivo.

The expressions of Beclin-1 and Bcl-2 were also determined by Western blot in vivo (Fig. 2) to calculate the ratio of beclin-1 to bcl-2 levels, which has been demonstrated to be of significance in the regulation of autophagy [3]. Our study showed that P188 significantly increased the Beclin-1/ Bcl-2 ratio in the injured cortex (Fig. 3A) and hippocampus (Fig. 3B).

\subsection{The results of scratch test and immunofluorescence assay}

To further confirm whether the autophagy is involved in the post-TBI recovery in vitro, the scratch test and LC3 immunofluorescence assay were performed. In scratch test, the wound healing rate significantly improved at $12 \mathrm{~h}$ and $24 \mathrm{~h}$ in P188 group (Fig. 4). The formation of LC3 puncta, representing autophagosomes, was extensive in cells exposed to P188 at $24 \mathrm{~h}$ after cell stretch-injury versus the injured groups in vitro (Fig. 5A, B). 


\section{Discussion}

With the development of industry and increasing traffic accidents, TBI has been a life threat worldwide and a burden to the health care system. Researchers are contributing to the prevention of traffic accidents and the therapeutic strategies for trauma. However, there is a paucity of information regarding the neuroprotective effects of P188 on the events secondary to TBI. Autophagy is a fundamental cellular homeostatic mechanism, whereby cells autodigest proteins, lipids and organelles of their cytoplasm for removal or turnover. It is a highly regulated mechanism [3]. Recently, our laboratory reported that using cortical neuron cultures, P188 was shown to protect neurons from trauma induced mitochondrial and lysosomal membrane permeabilization damage $[1,6]$. Therefore, we presumed that P188 may play an important role in the secondary injury after TBI through regulating autophagy.

We investigated the effect of P188 in autophagy following TBI both in vitro and in vivo, in which autophagy was activated subsequent to P188 treatment. P188 can be directly embedded in the cell membrane lipid layer in vitro and restore plasma membrane integrity in multiple cells $[8,16]$. Recent studies have shown that autophagy is increased after TBI [4]. In our scratch test, the PC-12 cells wound healing rate also was significantly increased at $12 \mathrm{~h}$ and $24 \mathrm{~h}$ with P188 administration in vitro. We thus aimed to investigate whether the autophagy was regulated by P188, and the neuroprotective effects [1] of P188 were due to its influence on autophagy.

LC3 is one of the gold-standard markers for the study of autophagy induction, with the conversion of the LC3-I protein to LC3-II well documented to be a hallmark [3]. Our results demonstrated that, in vivo, the conversion of LC3-I to LC3-II significantly enhanced in P188 treatment group (in both the injured cortex and injured hippocampus) after TBI versus the saline or sham groups. For further confirming the LC3 results, we also performed PC-12 cells experiments in vitro. The P188 post-treatment significantly increased the formation of LC3 puncta at $24 \mathrm{~h}$ after cell stretch-injury in vitro. All these results confirmed that autophagy was significantly 
activated by P188 after TBI.

Beclin-1 is an essential partner in the onset and activating autophagy, and researchers usually use it as a way to monitor autophagy. However, Beclin-1 can be inhibited by binding to the Bcl-2 protein, and autophagy is induced by the release of Beclin-1 from Bcl-2 proteins [3]. Beclin-1 and Bcl-2 are identified to be crucial proteins in apopotosis and autophagy, and their ratio is key indicator of the status of autophagy [11, 12], with a higher ratio indicating enhanced autophagy $[11,12]$. Additionally, without increasing the ratio of Beclin-1/Bcl-2, certain autophagy inducers (i.e. hypoxia, starvation, etc.) can also disrupt the binding of Beclin-1 to Bcl-2, thereby releasing the non-binding Beclin-1 to induce autophagy, leading to activation of signaling pathways such as JNK1-mediated phosphorylation of Bcl-2 [17]. In our experiment, the Beclin-1/Bcl-2 ratios in the P188 group (both of injured cortex and injured hippocampus) dramatically increased following TBI compared to saline mice. In addition, Beclin-1 can form a multi-protein complex that includes class III phosphatidylinositol-3-kinase (PI3K), also known as hVps34, which increased significantly upon cellular stimulation, thereby regulating the formation of nascent autophagosomes [20].

In addition to LC3, p62 is considered to be an autophagy marker, at least in certain settings [3]. The p62 protein serves as a link between LC3 and ubiquitinated substrates It is reported that the inhibition of autophagy correlates with upregulated expression of p62 [3], suggesting that the consistency of this protein levels reflects the autophagy status. In our study, compared with saline groups, we found that the P62 was decreased from $12 \mathrm{~h}$ to $48 \mathrm{~h}$ after TBI in vivo, the results indicated that the autophagy was activated by TBI. However, P188 post-treatment further down-regulated the p62 expression from $12 \mathrm{~h}$ to $48 \mathrm{~h}$ post TBI in vivo, indicating a more powerful autophagy was induced by P188 after TBI. These results suggest a increased ability to degrade endogenous substrates for autophagy, wherein p62 was incorporated into the complete autophagosome and was degraded in autolysosomes.

All the above data imply that the autophagy was enhanced by P188 both in vivo and in vitro. Post-traumatic administration of luteolin reportedly protects mice from TBI by inducing autophagy [19], and stimulation of autophagy by rapamycin 
protected mice brain from damage and promoted functional recovery in a model of acute focal CNS lesion [15]. However, at present, autophagy is a double-bladed sword or even controversial. Increased autophagy could be beneficial or detrimental in various neuronal injury models [3]. Moreover, autophagy is reported to contribute to cell death and function deficits after TBI post-treatment with P188 [6, 7]. These diversity and controversy could be attributed to the distinctions in disease models. Moderate autophagy contributes to cellular homeostasis, whereas while increased activation of autophagy leads to cell death. The role of P188-induced autophagy in secondary injury after TBI still requires further studies.

Our previous experiments also demonstrated that P188 can notably attenuate TBI-induced neuronal death, lesion volume, brain edema, motor and cognitive dysfunction, and P188 suppressed apoptosis via extrinsic or intrinsic pathways and might serve as a potential drug for clinical applications [1]. However, several doubts still remain unaddressed regarding the capacity of the neuronal autophagic machinery after plasmalemmal resealing by P188 or the nature of the increased autophagy in TBI, which requires further exploration in this respect.

\section{Conclusion}

This study has demonstrated the role of P188 in plasmalemmal resealing, which activated autophagy after TBI both in vivo and in vitro. Nevertheless, the effects of the enhanced autophagy by P188 on the secondary injury after TBI still need further exploration.

\section{Acknowledgements}

The project was supported by the National High Technology Research Program of China (The 863 Program, SS2015AA020503), the National Science Foundation of China (No. 81302612, No. 81172911 and No. 81301039). We also appreciate the support from the Priority Academic Program Development of the Jiangsu Higher Education Institutions.

\section{Conflict of interest}

The authors report no conflicts of interest in this work. 


\section{References}

[1] H.J. Bao, T. Wang, M.Y. Zhang, R. Liu, D.K. Dai, Y.Q. Wang, L. Wang, L. Zhang, Y.Z. Gao, Z.H. Qin, X.P. Chen, L.Y. Tao, Poloxamer-188 attenuates TBI-induced blood-brain barrier damage leading to decreased brain edema and reduced cellular death, Neurochemical research 37 (2012) 2856-2867.

[2] W. Chu, M. Li, F. Li, R. Hu, Z. Chen, J. Lin, H. Feng, Immediate splenectomy down-regulates the MAPK-NF-kappaB signaling pathway in rat brain after severe traumatic brain injury, The journal of trauma and acute care surgery 74 (2013) 1446-1453.

[3] D.J. Klionsky, K. Abdelmohsen, A. Abe, et al, Guidelines for the use and interpretation of assays for monitoring autophagy (3rd edition), Autophagy 12 (2016) 1-222.

[4] Y. Lai, R.W. Hickey, Y. Chen, H. Bayir, M.L. Sullivan, C.T. Chu, P.M. Kochanek, C.E. Dixon, L.W. Jenkins, S.H. Graham, S.C. Watkins, R.S. Clark, Autophagy is increased after traumatic brain injury in mice and is partially inhibited by the antioxidant gamma-glutamylcysteinyl ethyl ester, Journal of cerebral blood flow and metabolism : official journal of the International Society of Cerebral Blood Flow and Metabolism 28 (2008) 540-550.

[5] J.M. Lee, S.J. Park, D.S. Im, Lysophosphatidylethanolamine increases intracellular $\mathrm{Ca}(2+)$ through LPA(1) in PC-12 neuronal cells, Biochemical and biophysical research communications 461 (2015) 378-382.

[6] C. Luo, Q. Li, Y. Gao, X. Shen, L. Ma, Q. Wu, Z. Wang, M. Zhang, Z. Zhao, X. Chen, L. Tao, Poloxamer 188 Attenuates Cerebral Hypoxia/Ischemia Injury in Parallel with Preventing Mitochondrial Membrane Permeabilization and Autophagic Activation, Journal of molecular neuroscience : MN 56 (2015) 988-998.

[7] C.L. Luo, B.X. Li, Q.Q. Li, X.P. Chen, Y.X. Sun, H.J. Bao, D.K. Dai, Y.W. Shen, H.F. Xu, H. Ni, L. Wan, Z.H. Qin, L.Y. Tao, Z.Q. Zhao, Autophagy is involved in traumatic brain injury-induced cell death and contributes to functional outcome deficits in mice, Neuroscience 184 (2011) 54-63.

[8] J.J. Martindale, J.M. Metzger, Uncoupling of increased cellular oxidative stress and myocardial ischemia reperfusion injury by directed sarcolemma stabilization, Journal of molecular and cellular cardiology 67 (2014) 26-37.

[9] B.F. Miller, E. Keles, L. Tien, J. Zhang, D. Kaplan, E.H. Lo, M.J. Whalen, The pharmacokinetics and pharmacodynamics of Kollidon VA64 dissociate its protective effects from membrane resealing after controlled cortical impact in mice, Journal of cerebral blood flow and metabolism : official journal of the International Society of Cerebral Blood Flow and Metabolism 34 (2014) 1347-1353.

[10] N. Mizushima, T. Yoshimori, B. Levine, Methods in mammalian autophagy research, Cell 140 (2010) 313-326.

[11] S. Pattingre, A. Tassa, X. Qu, R. Garuti, X.H. Liang, N. Mizushima, M. Packer, M.D. Schneider, B. Levine, Bcl-2 antiapoptotic proteins inhibit Beclin 1-dependent autophagy, Cell 122 (2005) 927-939.

[12] R.C. Russell, H.X. Yuan, K.L. Guan, Autophagy regulation by nutrient signaling, Cell research 24 (2014) 42-57.

[13] G. Serbest, J. Horwitz, K. Barbee, The effect of poloxamer-188 on neuronal cell recovery 
from mechanical injury, J Neurotrauma 22 (2005) 119-132.

[14] S.S. Shin, E.R. Bray, C.Q. Zhang, C.E. Dixon, Traumatic brain injury reduces striatal tyrosine hydroxylase activity and potassium-evoked dopamine release in rats, Brain Res 1369 (2011) 208-215.

[15] M.T. Viscomi, M. D'Amelio, V. Cavallucci, L. Latini, E. Bisicchia, F. Nazio, F. Fanelli, M. Maccarrone, S. Moreno, F. Cecconi, M. Molinari, Stimulation of autophagy by rapamycin protects neurons from remote degeneration after acute focal brain damage, Autophagy 8 (2012) 222-235.

[16] T. Wang, X. Chen, Z. Wang, M. Zhang, H. Meng, Y. Gao, B. Luo, L. Tao, Y. Chen, Poloxamer-188 can attenuate blood-brain barrier damage to exert neuroprotective effect in mice intracerebral hemorrhage model, Journal of molecular neuroscience : MN 55 (2015) 240-250.

[17] Y. Wei, S. Pattingre, S. Sinha, M. Bassik, B. Levine, JNK1-mediated phosphorylation of Bcl-2 regulates starvation-induced autophagy, Molecular cell 30 (2008) 678-688.

[18] M.J. Whalen, T. Dalkara, Z. You, J. Qiu, D. Bermpohl, N. Mehta, B. Suter, P.G. Bhide, E.H. Lo, M. Ericsson, Acute plasmalemma permeability and protracted clearance of injured cells after controlled cortical impact in mice, Journal of Cerebral Blood Flow \& Metabolism 28 (2008) 490-505.

[19] J. Xu, H. Wang, X. Lu, K. Ding, L. Zhang, J. He, W. Wei, Y. Wu, Posttraumatic administration of luteolin protects mice from traumatic brain injury: implication of autophagy and inflammation, Brain Res 1582 (2014) 237-246.

[20] Y. Zhong, Q.J. Wang, X. Li, Y. Yan, J.M. Backer, B.T. Chait, N. Heintz, Z. Yue, Distinct regulation of autophagic activity by $\operatorname{Atg} 14 \mathrm{~L}$ and Rubicon associated with Beclin 1-phosphatidylinositol-3-kinase complex, Nature cell biology 11 (2009) 468-476.

\section{Figure legends}

Fig. 1 P188 acutely further increased the LC3 and down-regulated P62 levels in the cortex and hippocampus after TBI. (A, D) Proteins from the cortex and hippocampus in each group were separated using SDS-PAGE, the LC3 and P62 protein levels were measured using western blotting. (B, C, E, F) Quantitative analysis of LC3 and P62 in the cortex and hippocampus. The optical density of each protein band was determined using Quantity One (Bio-Rid) and normalized to the loading control (GAPDH). Data were expressed as the mean \pm SE $(n=6) .{ }^{*} P<0.05$ and $* * P<0.01$ vs. saline group at the same time point; ${ }^{\#} P<$ $0.05,{ }^{\# \#} P<0.01$ vs. sham group.

Fig. 2 Analysis of Beclin-1 and Bcl-2 expression in cortex and hippocampus by western blotting. (A, D) Proteins from the TBI and sham-operated cortex and hippocampus were separated on SDS-PAGE. (B, C, E, F) Quantitative analysis of Beclin-1 and Bcl-2 in cortex and hippocampus; optical densities of respective protein bands were analyzed with Quantity 
One (Bio-Rid) and normalized with loading control (GAPDH). Data were expressed as mean \pm SE $(n=6) . * P<0.05$ and $* * P<0.01$ vs. saline group at the same time point; and ${ }^{\#} P$ $<0.05,{ }^{\#} P<0.01$ vs. sham group.

Fig. 3 The ratios of Beclin-1/Bcl-2 in injured cortex and hippocampus were significantly increased by P188 after TBI. (A) The ratio of Beclin-1/Bcl-2 in injured cortex. (B) The ratio of Beclin-1/Bcl-2 in injured hippocampus. Data were expressed as mean \pm SE $(n=6) . * P<$ 0.05 and $* * P<0.01$ versus saline group; ${ }^{\# \#} P<0.01$ versus sham group.

Fig. 4 In scratch test, compared with the control groups, the wound healing rates of PC-12 cells were significantly improved at $12 \mathrm{~h}$ and $24 \mathrm{~h}$ in P188 groups $(n=3)$.

Fig. 5 (A) P188 up-regulated the LC3 puncta (autophagosomes) in PC-12 cells in vitro $24 \mathrm{~h}$ after cell stretch-injury. (B) The corresponding linear diagram of LC3 staining. Data were expressed as mean $\pm \mathrm{SE}(n=3)$. ${ }^{* *} P<0.01$ versus saline group; ${ }^{\# \#} P<0.01$ versus sham group. 
A Injured cortex

Saline

P188

Sham $1 \quad 6 \quad 12 \quad 24 \quad 48 \quad 1 \quad 6 \quad 12 \quad 24 \quad 48 h$ $1 . \mathrm{C} 3-\mathrm{I}=$

$1 . \mathrm{C} 3-\mathrm{II}$

GAPDH

Injured Hippocampus

$$
\text { LC. } 3-1=
$$$$
\text { LC3-II }
$$

GAPDH
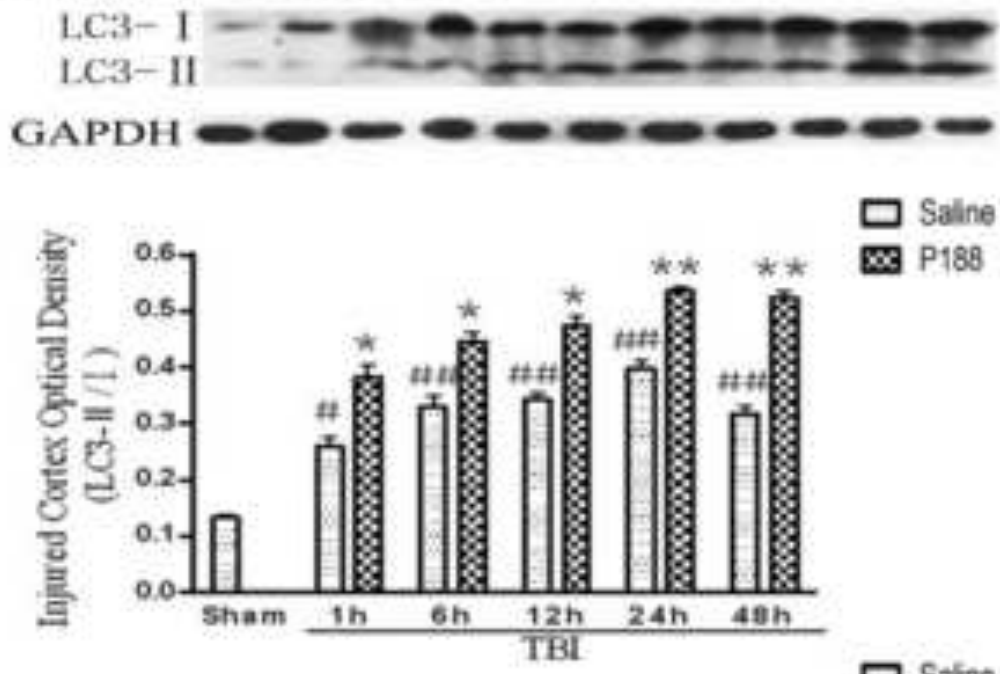

C

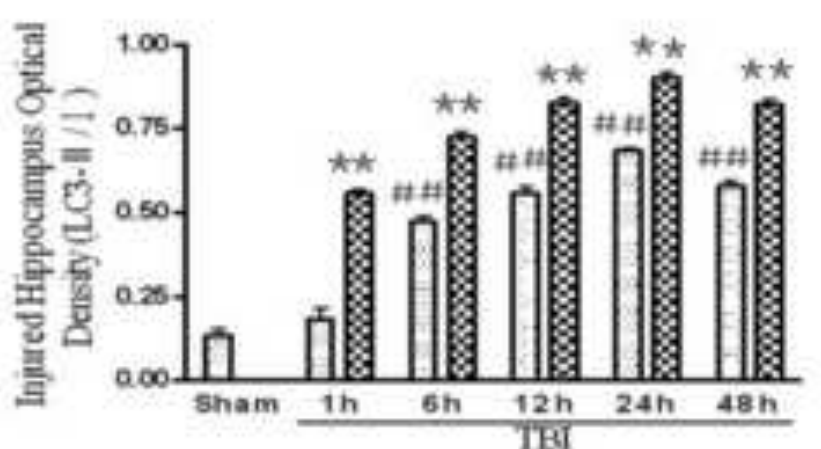
P188

ㅁ Saline P 188
D Injured cortex

$$
\text { Sham } \begin{array}{lllll}
\hline \quad 6122448 & 16122448 \mathrm{~h}
\end{array}
$$

P62

GAPDH $-0-0-0$

Injured Hippocampus

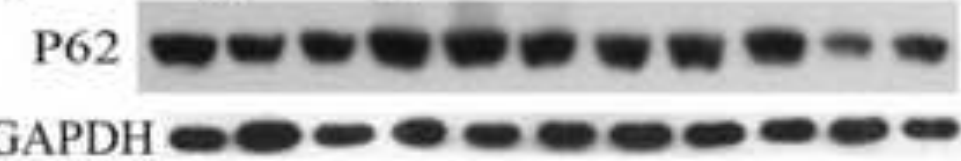

GAPDH

$\mathrm{E}$ 랄 ${ }^{1.5} 7 \mathrm{H}$ 口
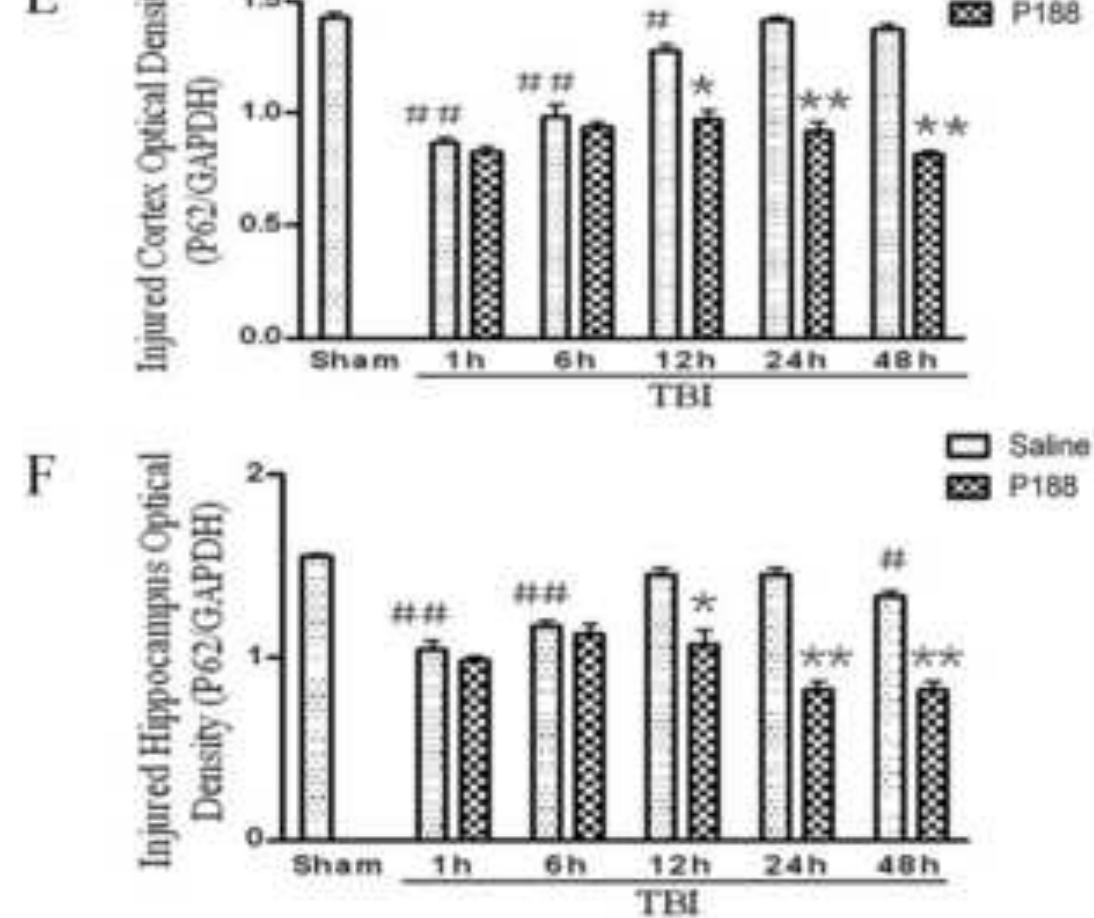
A Injured cortex

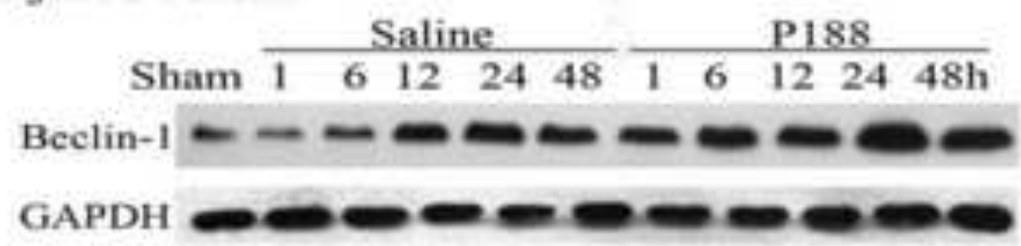

Injured Hippocampus

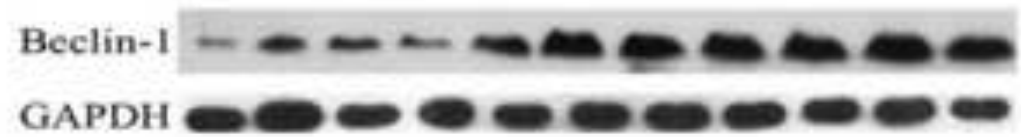

B

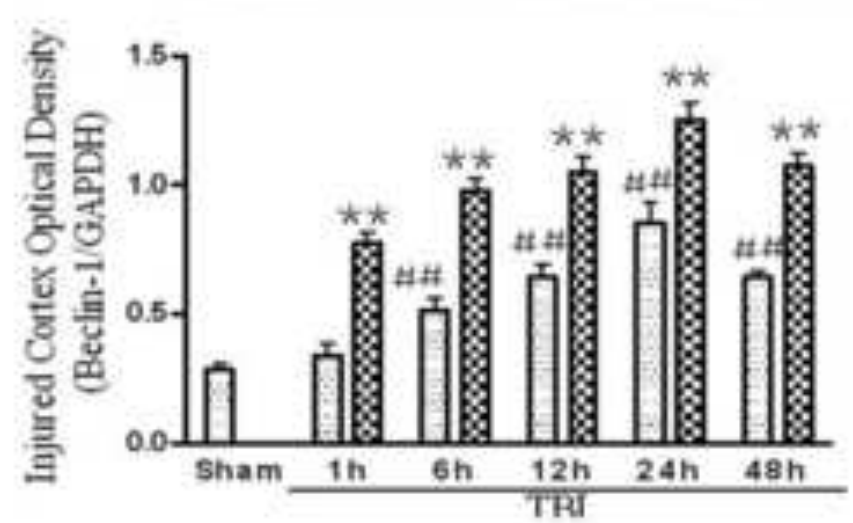

C

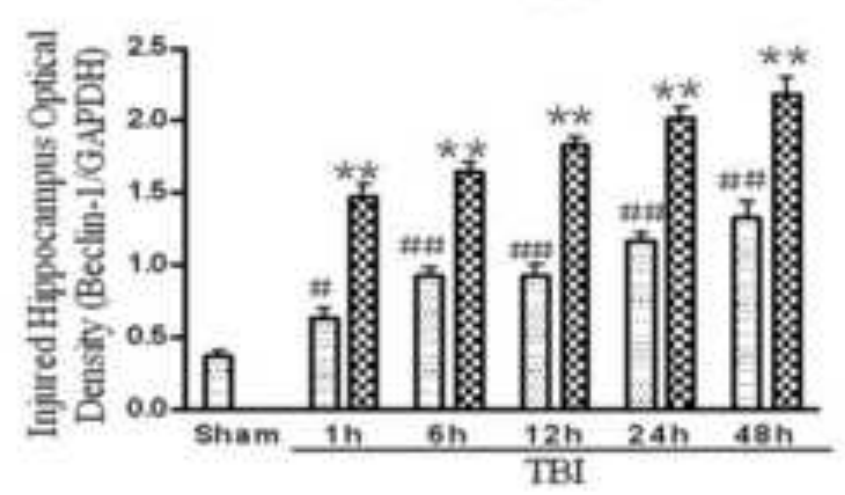

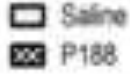

D

Injured cortex

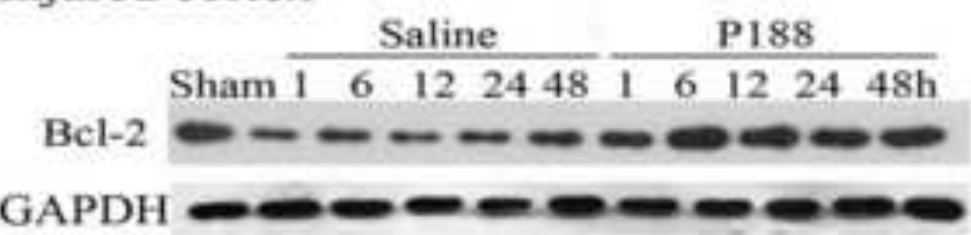

Injured Hippocampus

$$
\mathrm{Bcl}-2-\cdots-\cdots-\cdots
$$

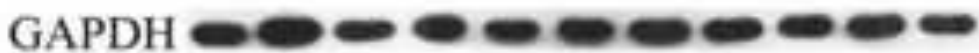

E
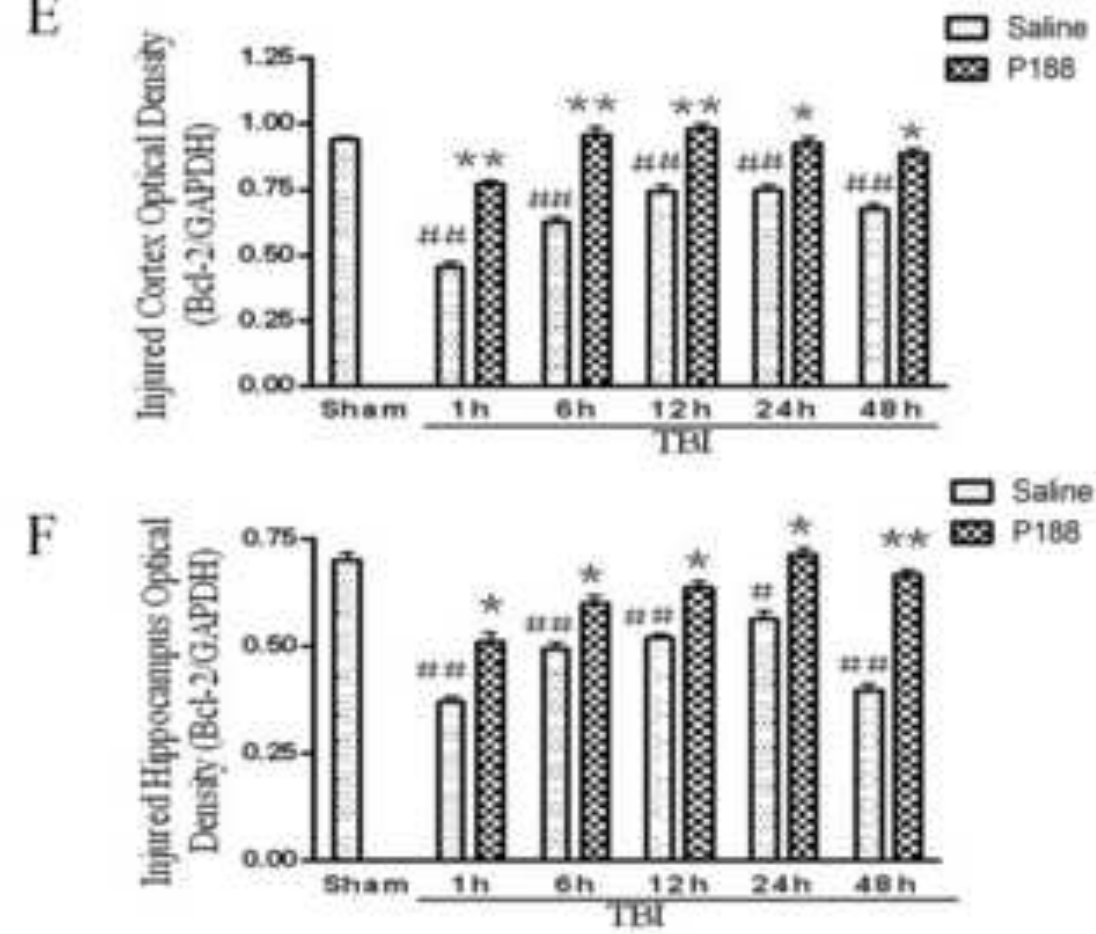
A

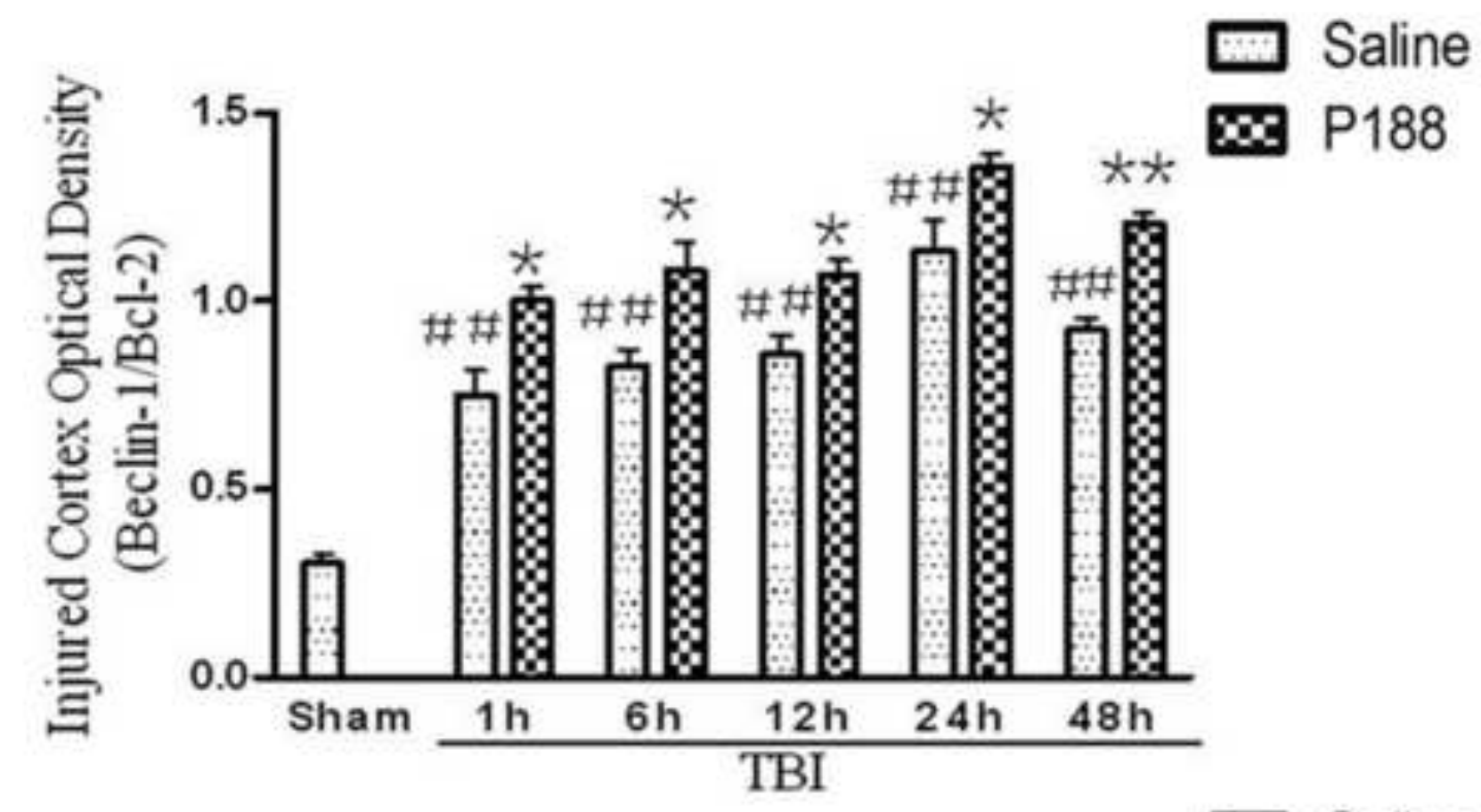

B

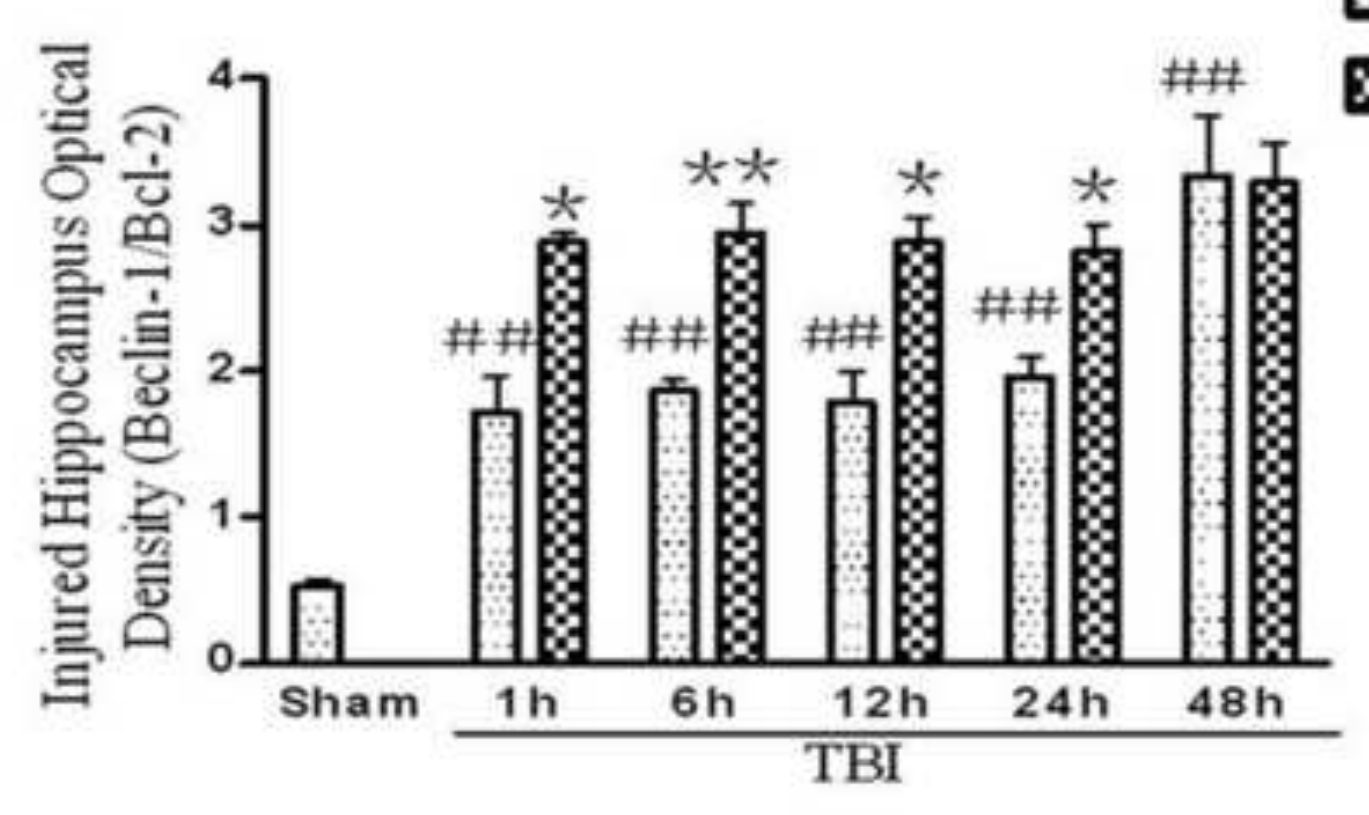

E. Saline

(x) P188 
A
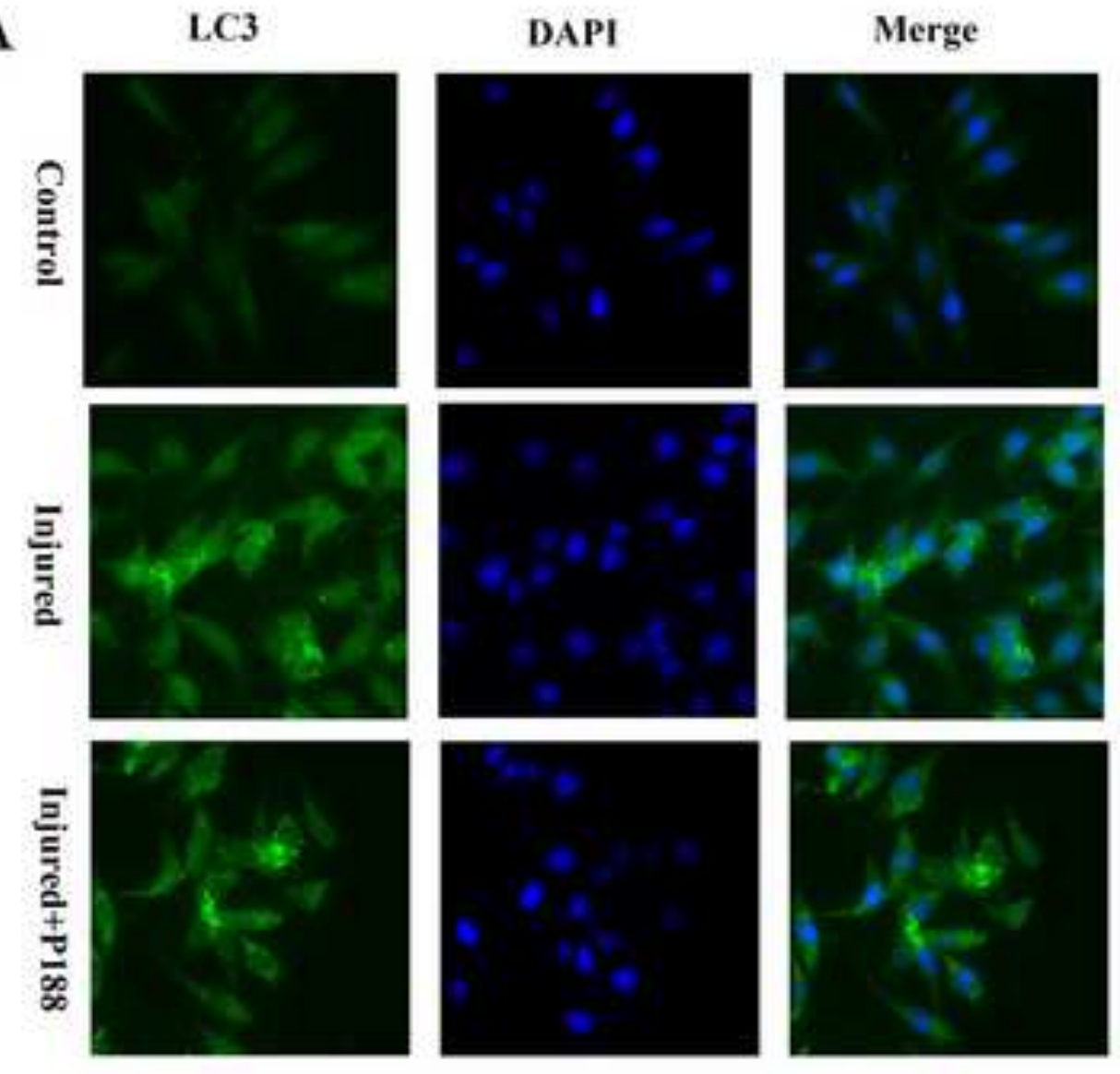

DAPI
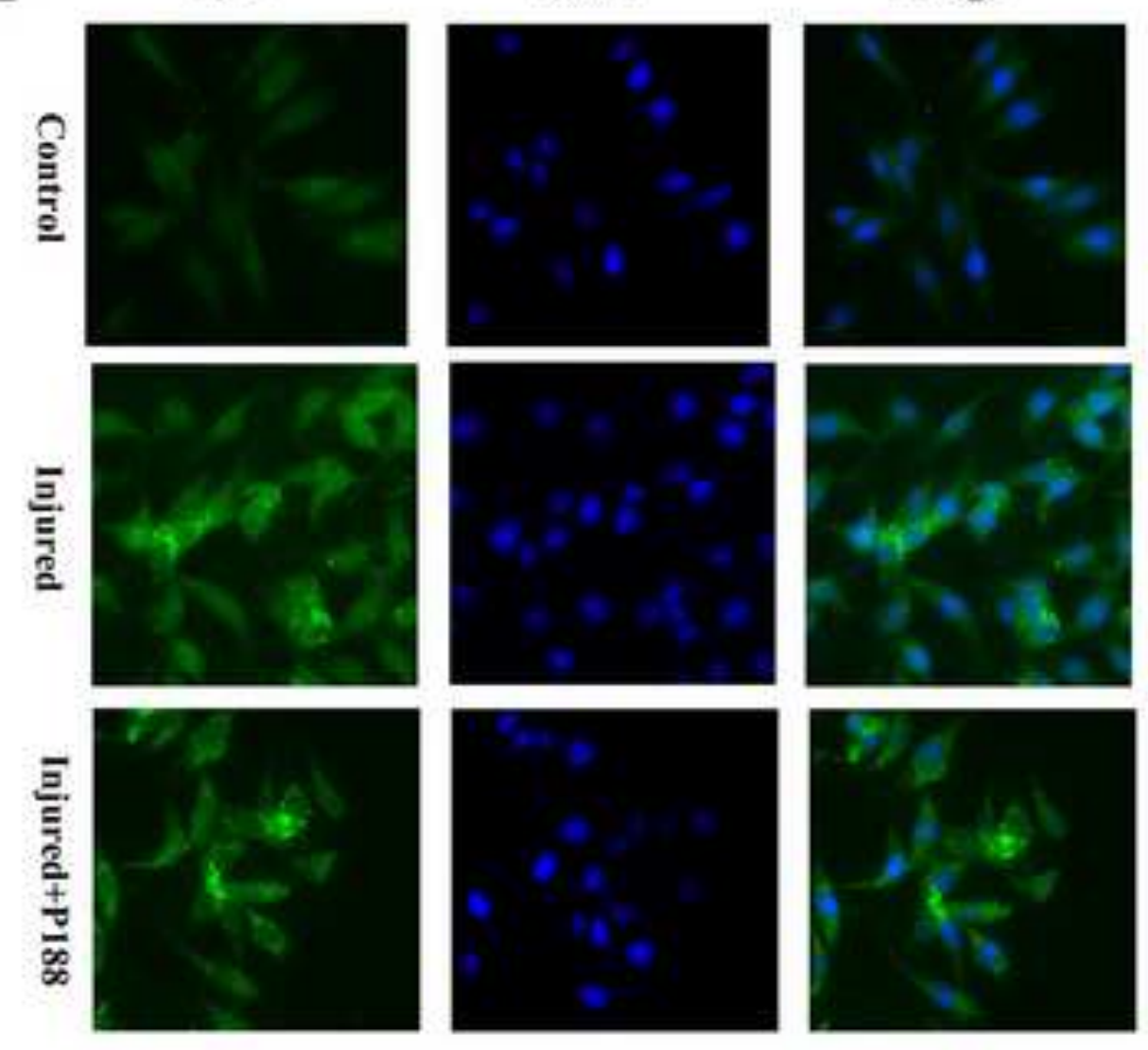

Merge
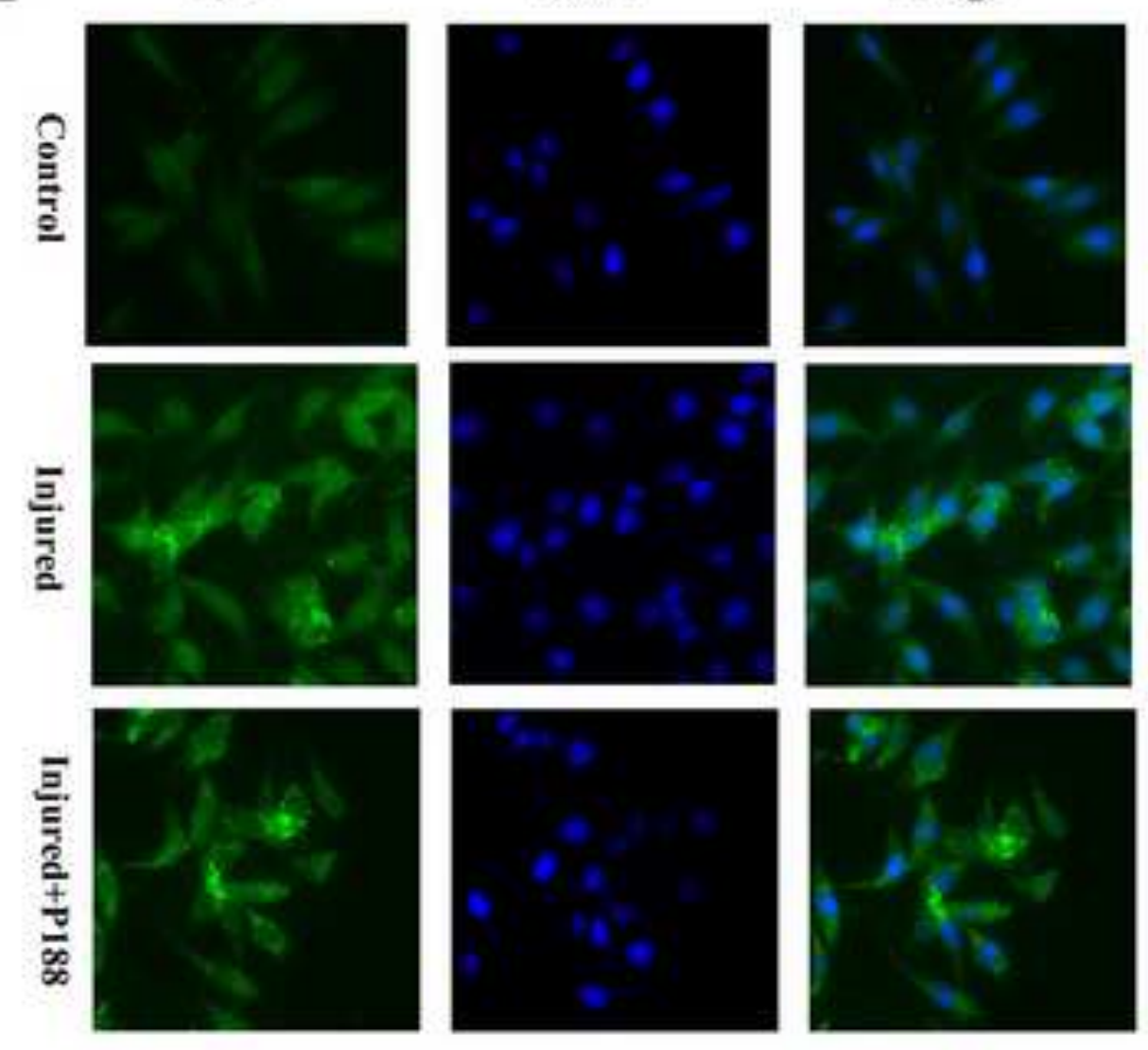

B

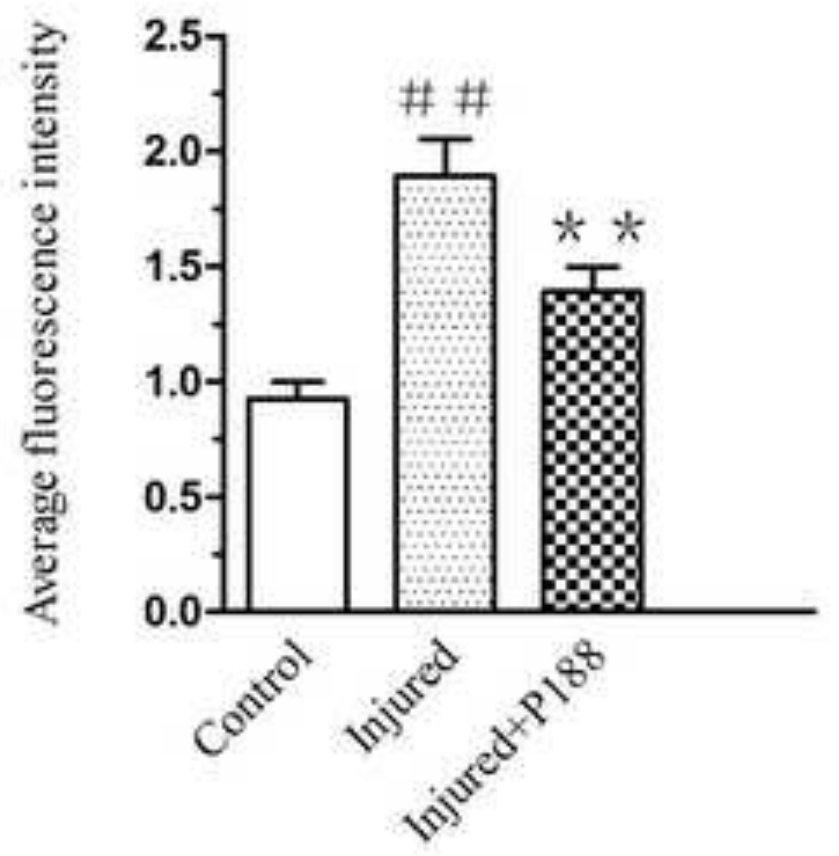


